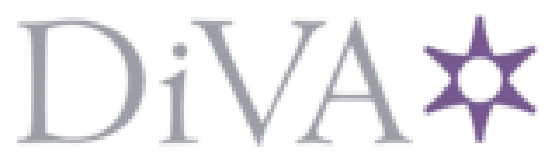

http://www.diva-portal.org

This is the published version of a paper published in Physical Review E. Statistical, Nonlinear, and Soft Matter Physics.

Citation for the original published paper (version of record):

Mattsson, A., Uesaka, T. (2015)

Time-dependent statistical failure of fiber networks.

Physical Review E. Statistical, Nonlinear, and Soft Matter Physics, 92(4): 042158

http://dx.doi.org/10.1103/PhysRevE.92.042158

Access to the published version may require subscription.

N.B. When citing this work, cite the original published paper.

Permanent link to this version:

http://urn.kb.se/resolve?urn=urn:nbn:se:miun:diva-26260 


\title{
Time-dependent statistical failure of fiber networks
}

\author{
Amanda Mattsson* and Tetsu Uesaka ${ }^{\dagger}$ \\ Department of Chemical Engineering, Mid Sweden University, Sundsvall, Sweden 85170
}

(Received 26 June 2015; published 28 October 2015)

\begin{abstract}
Numerical simulations of time-dependent stochastic failure of fiber network have been performed by using a central-force, triangular lattice model. This two-dimensional (2D) network can be seen as the next level of structural hierarchy to fiber bundles, which have been investigated for many years both theoretically and numerically. Unlike fiber bundle models, the load sharing of the fiber network is determined by the network mechanics rather than a preassigned rule, and its failure is defined as the point of avalanche rather than the total fiber failure. We have assumed that the fiber in the network follows Coleman's probabilistic failure law [B. D. Coleman, J. Appl. Phys. 29, 968 (1958)] with the Weibull shape parameter $\beta=1$ (memory less fiber). Our interests are how the fiber-level probabilistic failure law is transformed into the one for the network and how the failure characteristics and disorders on the fiber level influence the network failure response. The simulation results showed that, with increasing the size of the network $(N)$, weakest-link scaling (WLS) appeared and each lifetime distribution at a given size approximately followed Weibull distribution. However, the scaling behavior of the mean and the Weibull shape parameter clearly deviate from what we can predict from the WLS of Weibull distribution. We have found that a characteristic distribution function has, in fact, a double exponential form, not Weibull form. Accordingly, for the 2D network system, Coleman's probabilistic failure law holds but only approximately. Comparing the fiber and network failure properties, we found that the network structure induces an increase of the load sensitivity factor $\rho$ (more brittle than fiber) and Weibull shape parameter $\beta$ (less uncertainty of lifetime). Superimposed disorders on the fiber level reduce all these properties for the network.
\end{abstract}

DOI: 10.1103/PhysRevE.92.042158

PACS number(s): 02.50.-r, 05.10.-a, 62.20.-x

\section{INTRODUCTION}

Statistical failures of materials have been attracting keen interest both in statistical physics and in reliability engineering. Particularly today, as society's focus moves onto sustainability and the effective use of materials and energy, and thus correcting overdesign situations by increasing reliability, are of prime importance on many engineering fronts. Figure 1 shows typical creep failures and corresponding frequency distributions of lifetime and static strength of linerboard (used for packaging construction) [1]. As compared with typical compressive strength, which shows almost deterministic failure, creep lifetime varies drastically_its coefficient of variation (COV) exceeds more than $100 \%$. This large variation of creep lifetime is quite universal and has been found also for many other materials (for example, Refs. [2-4]).

Perhaps the most important question in today's material design is how to describe and predict time-dependent statistical failures of materials for a general loading history. This question directly touches upon the following fundamental questions that have been discussed for many years in the area: the size scaling of lifetime distribution, the form of the distribution function, and the damage evolution law. Although good reviews are available for some of these topics (e.g., Refs. [2,5-7]), we will revisit the literature specifically from the point of addressing the above questions.

The earlier work by Coleman is probably the first formal, axiomatic development of the analytical theory of timedependent statistical failure [8,9]. The theory is based on three postulates: (1) weakest-link hypothesis, (2) a breakdown rule

\footnotetext{
*amanda.mattsson@miun.se

†tetsu.uesaka@miun.se
}

that describes the rate of breakdown (damage), and (3) a probabilistic failure condition using the damage variable. Based on these three postulates, he derived a cumulative distribution function of lifetime in a general form. This general formulation can be further specialized by introducing the power-law breakdown rule and the power-law form of the probabilistic failure condition, resulting in the most widely used forms of the distribution function of time-dependent statistical failure. Because of its hypotheses, this formulation was proposed as a model for fiber. Therefore, it has been used extensively as an almost default model for fiber in modeling fiber-bundle systems [2,6,10-14].

One of the postulates, weakest-link scaling (WLS), is intuitively obvious when one-dimensional elements are linked in series, such as "fibers." However, WLS does appear also in multidimensional systems for brittle and quasibrittle cases [7,15]. Qualitatively, it is explained that as damages grow and a number of damage clusters are created, one of such clusters, normally the biggest cluster, grows into a critical size, leading to avalanche failure of the entire system [5]. Such critical cluster must be large enough to trigger the avalanche but must be still sufficiently small as compared with a typical system size in order to ensure the independence of individual, hypothetical cells (or links) that contain cracks. Therefore, the above hypothesis implies that, first, there is a lower bound of the system size at which WLS emerges and, second, the system must be brittle in order for WLS to appear. This has been demonstrated in fiber-bundle models for both static and time-dependent failures. The degree of load sharing, either equal load sharing (ELS) or local load sharing (LLS), affects the emergence of WLS: The ELS case does not follow WLS [6,16-18]. Even in the LLS case, as will be described later, a parameter $\rho$ that controls damage evolution affects the scaling behavior. At higher $\rho$ values 
(a)

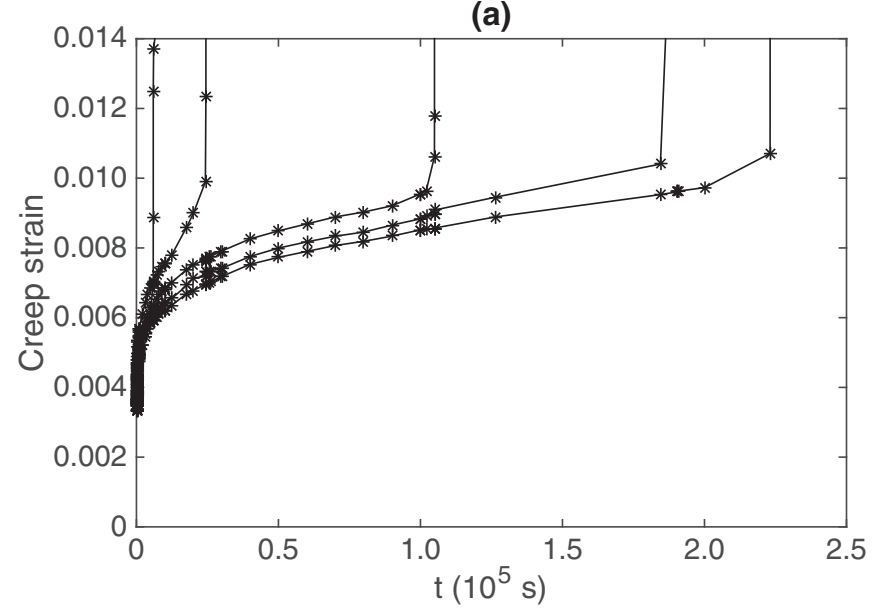

(b)

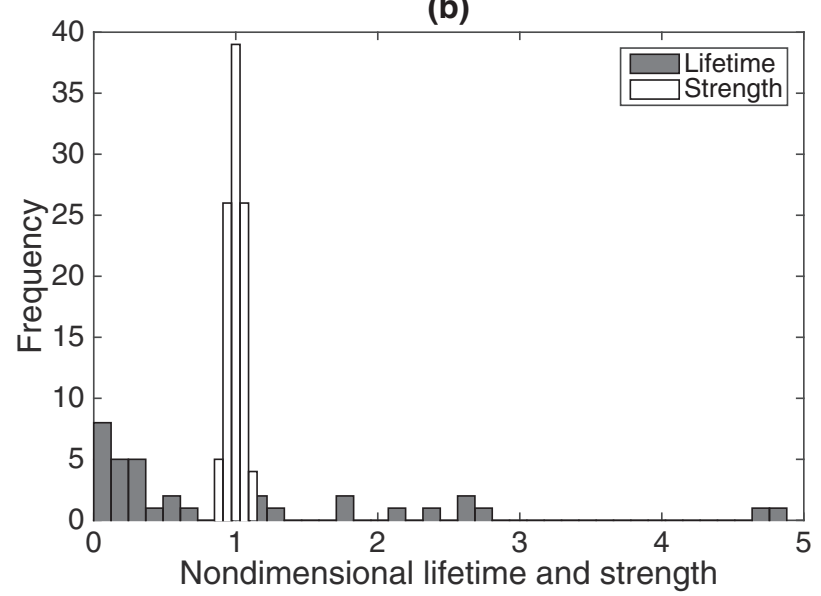

FIG. 1. (a) Typical creep compression curves and failures of linerboard and its (b) frequency distribution of lifetime and static strength [1]. The lifetime and strength are normalized by their means.

( $\gg 2$, brittle), LLS systems clearly shows WLS, but as $\rho$ becomes smaller, the size scaling of LLS systems approaches to that of ELS $[6,10,18]$. In addition, when load sharing and fiber failure are correlated, such as hierarchical fiber bundle models $[19,20]$, the scaling behavior deviates from WLS. Therefore, in a higher-dimensional system (2D and 3D), as load sharing is more extended (somewhat between ELS and LLS) and microstructures have long-range correlations, the emergence of WLS may not be automatic. This may pose a question in extending Coleman's model to higher-dimensional systems. It should also be noted that the experimental investigations of WLS is generally very difficult (except in the case of fibers), because of the needs for different pieces of equipment for testing specimens with grossly different sizes as well as the time required for performing a large number of repetitions. Even the numerical studies of WLS are limited to relatively simple systems, such as fiber bundles, because of high computational demands.

The breakdown rule defines the evolution of damages by taking into account loading history effects. The most commonly used breakdown rules are exponential and powerlaw forms. Because the exponential form originated from the earlier work on absolute reaction rate theory for creep failure [21], it has been argued in the area of experimental creep failure analyzes that the exponential form is a more faithful representation of the underlying molecular processes. However, Phoenix and Tierney showed that the power-law form is actually a better approximation of the stress-dependent thermal energy function [11]. One of the critical tests of this hypothesis is to examine the load dependence of mean creep lifetime [9]. So far the power-law form has not shown any obvious flaw, though the ranges of load tested are still limited (for example, Refs. [1-3]).

The most problematic may be the shape of lifetime distributions, specifically Weibull distribution. First, it depends on the size (and its geometry) of the system. The problem has been well recognized in the static strength distributions. Bazãnt discussed the deviation of the upper tail of static strength distribution from Weibull distribution in terms of the finitesize effect. He also predicted the transition from Gaussian to Weibull distribution in the lower tail. This transition behavior was described recently by introducing $\kappa$-Weibull distribution [22]. For a larger system where WLS is presumed to work, experimental data almost overwhelmingly showed Weibull distribution. Interestingly, numerical and analytical studies have shown otherwise. For example, for fiber-bundle models with $\rho \gg 1$ (more brittle) and a large system size where WLS appears, the distributions are clearly non-Weibull $[6,11]$. A microstructural model of damage evolution also suggested that double-exponential distribution is a better approximation in the low-probability tail [23]. This situation is also parallel to that of static strength distributions. However, it has been recognized that detecting non-Weibull behavior by experiments in a statistically significant manner is difficult, particularly in the probability range where experiments can be performed $[1,3,4,24]$. Nevertheless, the distribution shape in the lower tail (shorter lifetime) is still of great importance in the system reliability, as it is normally evaluated in the probability range in the order of $10^{-7}$ [7].

Another critical question is how the statistical failure properties of elements are transcended to the macroscopic material properties. Except the fiber bundle case, this question has been largely untouched. Recently unidirectional fiberreinforced composites are analyzed for both static strength and time-dependent failure [14,25].

In this paper we focus on a fiber network. The fiber network may be regarded as the next level structural hierarchy to fiber bundles. It may also be regarded as a paradigm of more general material systems, such as polycrystals, polymers, living tissues, paper, and nonwoven fabrics. We first review the general framework of Coleman's formulation, as it will be used later as a fiber model, and also it may be possible to use as a system model (under certain conditions). Second, we examine the postulates involved in Coleman's model by using numerical simulations of creep failure. The example of the fiber network is a triangular, central-force spring network. This model has been used in the past by many researchers to investigate various aspects of statistical failure under both static strength and creep test conditions (for example, Refs. [26,27]). In this paper we use this network structure specifically to examine the size scaling, the shapes of lifetime distributions, and the damage evolution law. Based on this, we further discuss the cases where 
there are uncertainties in the element properties and how they affect the lifetime distributions of the network.

It should be noted that this 2D system is distinct from the fiber bundle models in two aspects. First there is, obviously, no choice of load sharing rules (ELS and LLS). Load sharing is determined by mechanics. Second, in the network, the failure is defined as the point of avalanche (more precisely, the start of avalanche), not all element failures across the width, such as in the case of fiber bundle models. One of our interests is to see how the results from this extension are compared with those obtained in the fiber bundle models.

\section{THEORETICAL BACKGROUND}

Coleman's formulation of time-dependent statistical failure is based on three postulates. The first postulate is the WLS hypothesis, which is generally stated as

$$
1-F_{l}(t)=\left[1-F_{s}(t)\right]^{M},
$$

where $F_{l}(t)$ and $F_{s}(t)$ are the cumulative distribution functions of lifetime $t$ for the system of size $l$ and the element of size $s$, respectively, and $M=l / s$. The interpretation of the sizes $l$ and $s$ is obvious in the case of fiber, as the elements are linked in series in the system and the failure of the weakest element immediately triggers the entire system failure. However, in a $2 \mathrm{D}$ or $3 \mathrm{D}$ system, these size parameters require some consideration. For example, $s$ must be large enough to contain the largest cluster of damages (the existence of a lower bound of the size parameter $s$ [9]), and, at the same time, $l$ must be large enough to contain a number of the $s$ elements which are regarded as statistically independent in terms of damage evolution. Therefore, Eq. (1) is regarded as an asymptotic relation that may appear as $M$ increases. The $M$-independent $F_{s}(t)$ is often called the characteristic distribution function of a fictitious element [6]. The size of the fictitious element may be different depending on the anisotropy and also the loading direction in the $2 \mathrm{D}$ or $3 \mathrm{D}$ system, as will be seen in a later section.

The second postulate is a breakdown rule that defines how damage $\Omega(t)$ evolves as a function of time and other variables. The most general rule given by Coleman, the so-called Class $\mathrm{C}$ breakdown rule, states that the damage evolution rate depends on force (or stress), damage state, temperature, and their time derivatives in higher orders. However, the frequently used rule in the literature only concerns the force dependence in the following power-law form:

$$
\frac{d \Omega}{d t}=\kappa[f(t)]=c f(t)^{\rho},
$$

where $f(t)$ is a force (or stress) history and $c$ and $\rho$ are constants. $\rho$ determines the force-sensitivity of damage growth. This power-law form is often considered as a purely empirical relation, but Phoenix and Tierney pointed out that it is actually a good approximation of the thermal activation energy function and derived an expression for the constant $\rho$ as

$$
\rho=\frac{U_{0}}{k T},
$$

where $U_{0}$ is a fitting constant to the potential function, $k$ the Boltzmann constant, and $T$ the temperature [11]. As seen in its temperature dependence, $\rho$ captures the brittleness aspect of the failure. The damage evolution rule, Eq. (2), does not explicitly contain the dependence on the damage state $\Omega(t)$, unlike the corresponding rule in damage mechanics [28]. However, it can be easily shown that the above equation is an approximate case for the standard damage mechanics model when $\Omega$ is small (no large scale breakdown), so there is little stress-enhancing effect due to the presence of damages (mean-field effect). Therefore, the second postulate implicitly assumes that the extent of damages is still limited when the failure happens. (In the Appendix we derive the consequence of Kachanov's type breaking rule.)

The third postulate is a probabilistic fracture criterion:

$$
F_{s}(t)=\Psi[\Omega(t)],
$$

where the function $\Psi(\Omega)$ is a single-valued, positive monotonically increasing function of $\Omega$. The function may take any form as long as it satisfies this basic condition. However, considering the WLS hypothesis, one can expect that the function $\Psi$ only concerns small values of $\Omega$ when $\mathrm{M}$ in Eq. (1) is large. Coleman chose the following form as an approximation of the lower tail of the function $\Omega$ :

$$
\lim _{\Omega \rightarrow 0}=C \Omega^{\beta}+o\left(\Omega^{\beta}\right) .
$$

This power-law form, of course, can be derived from different routes from damage evolution models $[23,29]$ or percolation lattice models [30,31].

Using these three postulates, one obtains the expression for a cumulative distribution function of lifetime:

$$
\begin{aligned}
\lim _{M \rightarrow \infty} F_{l}(t) & =1-\exp \left\{-M C \Omega^{\beta}\right\} \\
& =1-\exp \left\{-A\left[\int_{s=0}^{t} f(s)^{\rho} d s\right]^{\beta}\right\},
\end{aligned}
$$

where $A=M C c^{\beta}$ absorbs the constants. Precisely speaking, this is a limiting distribution when $M \rightarrow \infty$. Therefore, in a finite-size range where WLS does not appear, the above equation may not hold. It should also be noted that, for a general loading history, the lifetime does not necessarily follow Weibull distribution; instead, the damage parameter $\Omega$ does follow Weibull, as seen in the first equation of Eq. (6). The damage parameter $\Omega$ plays the role of an internal time scale that takes into account loading history effects. In the case of creep condition, $f(t)=f_{c}$, then lifetime does follow Weibull distribution:

$$
F_{l}(t)=1-\exp \left\{-A f_{c}^{\rho \beta} t^{\beta}\right\}=1-\exp \left\{-\left(\frac{t}{t_{s}}\right)^{\beta}\right\},
$$

where $t_{s}=\left(f_{c}^{\rho} A^{1 / \beta}\right)^{-1}$. In this specific Weibull distribution, the load dependence appears only in the scale parameter $t_{s}$, not in the shape parameter $\beta$, and the scale parameter is inversely proportional to $f_{c}^{\rho}$. This load dependence can be used as one of the critical tests for a class of Coleman's model [4]. However, it is often difficult to perform such tests experimentally at lower stress levels (e.g., 50\% or lower) because of unrealistically long times required for break specimens. Numerical methods may be more appropriate for this purpose.

In numerical simulations, the hazardous function or failure rate function $h(t)$ is used instead of the cumulative distribution 
function (or probability density function):

$$
h(t)=\frac{F^{\prime}(T)}{1-F(t)}=A \beta f(t)^{\rho}\left[\int_{\xi=0}^{t} f(\xi)^{\rho} d \xi\right]^{\beta-1} .
$$

Here $h(t) d t$ represents a probability of failure between $t$ and $t+d t$ when the system survived up to time $t$. When $\beta=1$, the hazardous function depends only on the current value of load $f(t)$ and not on its loading history. In other words, such a system has no memory in the hazardous function. Because of the Malkov property, this model has been frequently used as a fiber model in many numerical studies. In our investigation we also use it as an element model in the triangular lattice system.

\section{MODEL ASSUMPTIONS}

\section{A. Network geometry}

We have used a two-dimensional network with a centralforce, triangular lattice (Fig. 2). The geometry of this model is, of course, highly simplified, but the model still retains essential network mechanics and also the same complex statistical failure properties as more realistic fiber network models.

Constant forces are applied at the top boundary, either tensile or compression, to simulate creep, and a tractionfree boundary condition is employed at the sides. Periodic boundary conditions, however, are not used in this study in order to avoid introducing artificial length scales to the failure phenomena of the system. In addition, the presence of edges is an important feature of real materials. The two upper rows are prevented from failure to avoid instability induced by large rotation of the failed elements. Each element has the same elastic modulus and the same length and diameter, though these can be easily varied. In the following we call each element fiber. The size of the network is described by $m$ (the number of stacks) and $n$ (the number of horizontal fibers), as seen in Fig. 2.

\section{B. Stochastic fiber lifetime model}

For individual fibers, we have used Coleman's fiber model with $\beta=1$ (memory-less fiber). We rewrite Eq. (6) for the case of $\beta=1$ in a nondimensional form:

$$
F_{f}(\tilde{t})=1-\exp \left\{-\int_{\tilde{s}=0}^{\tilde{t}}\left[\frac{T(\tilde{s})}{T_{c}}\right]^{\rho} d \tilde{s}\right\},
$$

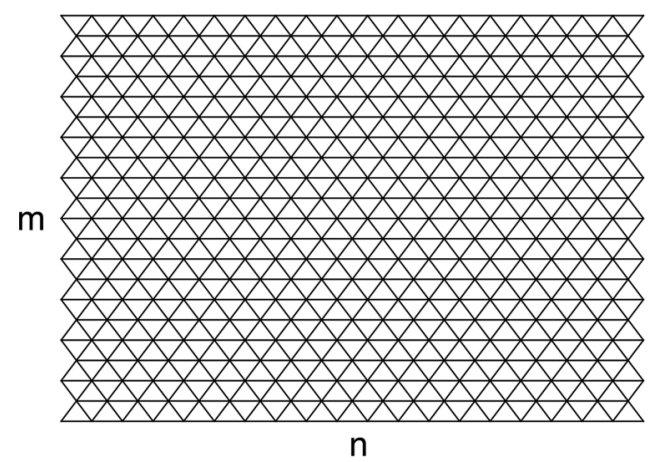

FIG. 2. Schematic of the central-force, triangular lattice used in the simulations. where $T(\tilde{s})$ is the force history applied to an individual fiber, nondimensional time $\tilde{t}=t / t_{0}$, and $t_{0}=\left(A T_{c}^{\rho}\right)^{-1}$. If $t_{0}$ is taken as a unit time, then $T_{c}$ is interpreted as characteristic strength of fiber (i.e., the load at which the fiber fails with the probability of $1-1 / e=0.6321$ before the unit time is reached). In the following, we suppress $\sim$ in $\tilde{t}$ but with the understanding that it refers to nondimensional time.

We first apply a dead load at the top boundary and then calculate forces created in individual fibers. Using these forces of individual fibers, we generate random numbers according to Eq. (9) with the constant loading history (i.e., exponential distribution). This determines lifetimes of individual fibers. The first failure occurs at the fiber with the shortest lifetime among the whole generated lifetimes. We set the modulus for this failed fiber to zero (or a small number) and then recalculate the force in each fiber for a new state of mechanical equilibrium. At this point, the survived fibers are given new force states in a stepwise manner. This means that the original estimate of lifetimes for the survived fibers must be updated with the new loading history. This is done by solving Eq. (9) for a new lifetime with the new loading history, and one can obtain a simple updating formula for lifetime. For example, the updating formula after the first fiber break is given by

$$
t_{B 2}-t_{1}=\left[\frac{T(0)}{T\left(t_{1}\right)}\right]^{\rho}\left(t_{B 1}-t_{1}\right)
$$

where $t_{1}$ is the time of the first fiber failure in the system and $t_{B 1}$ and $t_{B 2}$ are the first and the second estimate of lifetime of a survived fiber. $T(0)$ and $T\left(t_{1}\right)$ are the forces of the survived fiber before and after the first fiber failure. The minimum of the updated lifetimes of all survived fibers is then found, and this determines the second fiber failure. This process continues until the avalanche failure of the system takes place.

This algorithm more faithfully reproduces fiber-breaking processes than the standard algorithm used for triangular lattice models (e.g., Refs. [23,26,27,32]). It should be noted that the random number generation [following exponential distribution in Eq. (9)] is made only once, and the subsequent fiber breaking steps are completely deterministic as dictated by micromechanics. This algorithm is in the same spirit of those used for fiber bundle models (e.g., Ref. [20]).

The avalanche is defined when the process meets the following two conditions: One is when the ratio of the current rate of the creep strain to the initial rate exceeds a certain value $r$. The other is that such high creep rate continues for a certain number of consecutive time steps $k$. Within the parameter space tested, we found that $r=100$ and $k=5$ can consistently detect the initiation of avalanche leading to final failure.

The force equilibrium of the triangular lattice system was obtained by using a truss analysis code for solving 2D and 3D structures [33]. To accelerate the calculations, and enable simulations of larger networks, the code was modified by introducing a MATLAB sparse matrix library.

The default set values used for the fibers (truss elements) in the model are controlled in order to limit overall deformation within a small strain (geometrically linear) range. Denoting characteristic strength as $T_{c}$, elastic modulus $E_{f}$, crosssectional area $A$, and the applied load at each node $S_{0}$, we used $T_{c} / S_{0}=10 / 3$ and $E_{f} A / S_{0}=500 / 3$ as a default. The 
(a)

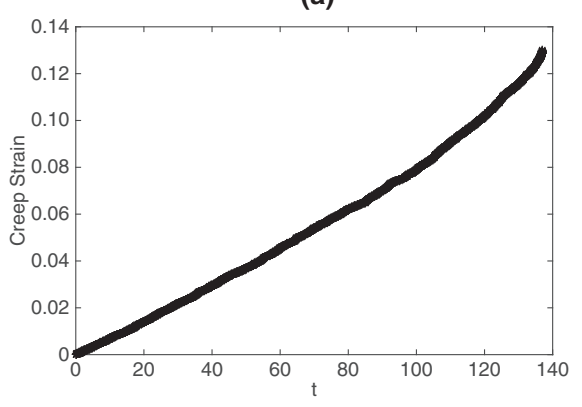

(b)

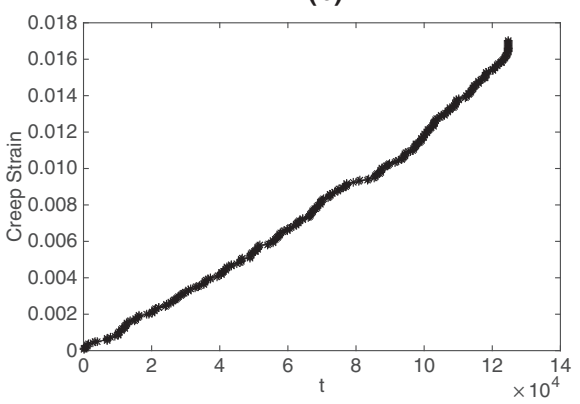

(c)

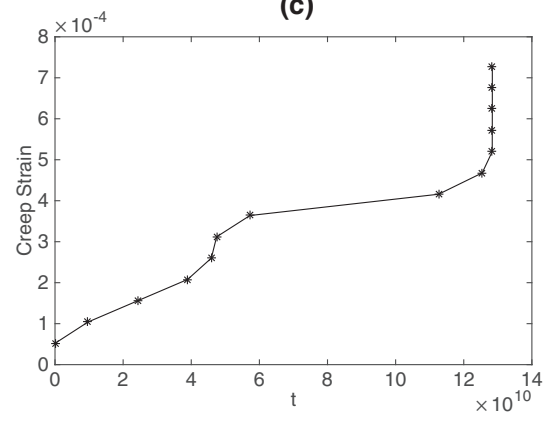

FIG. 3. Creep curves obtained from the simulations; each marked point represents an individual fiber break. From left to right: (a) $\rho=5$, (b) $\rho=10$, and (c) $\rho=20$.

default value of $\rho$ is 10 , and it later varied from 5 to 400 to cover the quasibrittle-to-brittle failure range.

\section{NUMERICAL RESULTS}

\section{A. Typical creep curves and damage evolution}

We begin with looking at qualitative features of the simulation results. Figure 3 shows typical creep curves obtained from the simulations. Each marked point corresponds to an individual fiber break. (Note that since the strain increments are created at the time of fiber failure, real creep strain is a stepwise function.) Results for three different values of $\rho$ are presented: [Fig. 3(a)] $\rho=5$, [Fig. 3(b)] $\rho=10$, and [Fig. 3(c)] $\rho=20$. The size of the system is set to $200 \times 200$. (We will discuss the size effect later.) Since these creep curves represent a sequence of elastic failures of fibers, the shapes are rather erratic unlike typical creep curves for viscoelastic body. As expected, with increasing $\rho$, the system becomes more brittle, and less fiber breaks are required before the avalanche type failure. The range of $\rho$ values used here already exceeds the threshold $(\rho=2)$ that was defined by Curtin for the tough-brittle failure transition [23].

The corresponding damage evolutions are shown in Fig. 4. At lower values of $\rho$, a large-scale destruction occurs in the entire structure, and critical clusters of damages appears only in a diffused way. However, at $\rho=20$, one can easily identify such a critical crack just before the avalanche failure. We found that, for high values of $\rho$, the stress intensity in the edges makes the lattice more vulnerable to create critical clusters leading to failure in those regions. In the case of fiber bundle systems, the critical cluster of contiguous damages, so-called $k^{*}$ crack, is used to obtain an upper bound of the lifetime distribution function [11,12]. In the case of the 2D system, however, the size and geometry of such critical cluster is more variable and diffused, as the cluster grows both in the loading and its perpendicular directions depending on underlying disorders in the structures.

\section{B. Lifetime distribution and size effect}

We first investigate lifetime distributions and size effects by changing the length $(m)$ and width $(n)$ of the system. Creep failure simulations were performed 1000 times for each size. This number of repeats was found earlier to be sufficient to detect non-Weibull behavior in a finite-size range [6,34]. The distributions are plotted in the Weibull format in order to examine a fit to Weibull distribution as well as the emergence of WLS.

Figure 5 shows the effect of increasing length $m$ with constant width $n=10$. After vertically shifting the curves of different length systems by $\ln (N)$, almost all curves collapsed into one, indicating WLS. Weakest-link scaling in the length direction may seem obvious by considering a series link of fibers, but for random fiber networks, WLS does not clearly appear even in the length (loading) direction [35]. The collapsed curve had a slight feature of a concave function, which is similar to those seen in fiber bundle models [6]. Note that the width $n=10$ is still in the finite-size range, as seen below.

The effect of increasing width $n$ (with constant length $m$ ) is shown in Fig. 6. In this case, the collapse of the curves for different widths appeared much later $(n \sim 200)$ than the previous case of the length direction. Although it is difficult (a)

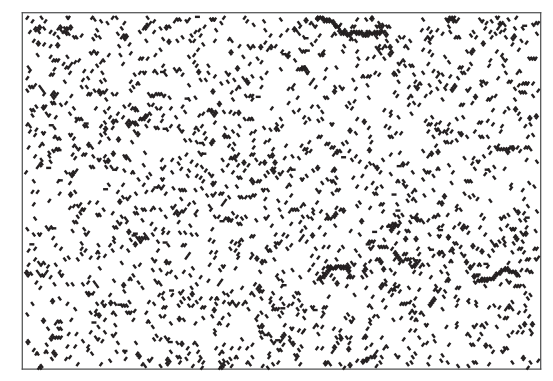

(b)

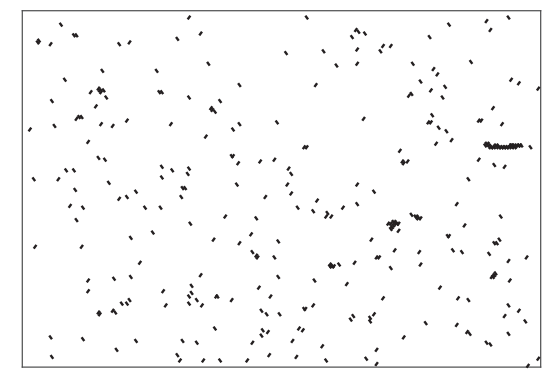

(c)

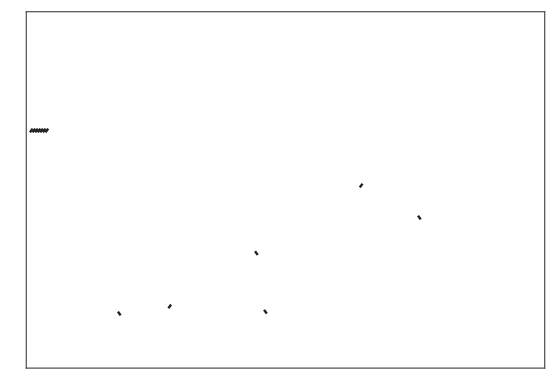

FIG. 4. Damage evolutions corresponding to the creep curves shown in Fig. 3. From left to right: (a) $\rho=5$, (b) $\rho=10$, and (c) $\rho=20$. 


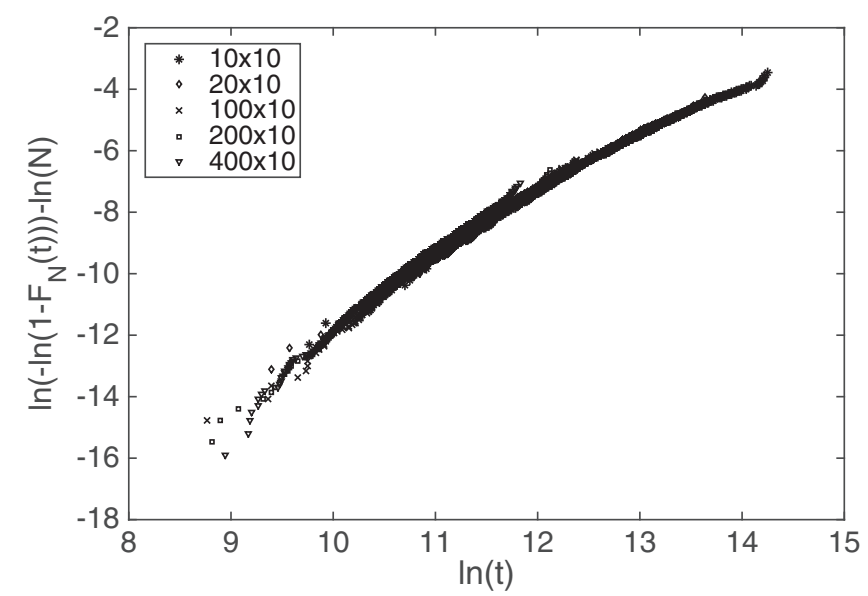

FIG. 5. Lifetime distributions for different sizes of the length of the lattice plotted in a Weibull format with a scaling term.

to compare the $1 \mathrm{D}$ and $2 \mathrm{D}$ models on the same basis, the emergence of WLS seems to be more rapid for the fiber bundle model using LLS than the $2 \mathrm{D}$ case with $\rho=10$ and $\beta=1$. This may be because load sharing in the $2 \mathrm{D}$ case is obviously much extended than the LLS.

The same plots are shown when length $m$ and width $n$ are changed simultaneously (Fig. 7). Similarly to the case of width scaling, the WLS trend appeared when the width approaches 200 or 400 .

Since the distribution curves for individual sizes are approximately linear, i.e., Weibull distribution, we plotted the slopes of the curves in Fig. 8 as a function of the total number of fibers $N$. With increasing the size, the slope increased almost linearly within the range of $N$ tested, instead of the slope approaching to a constant value. In other words, the distribution does not seem to have the Weibull lower tail (constant slope) suggested by Bažant [7].

Curtin and Scher $[23,26]$ developed an analytical model for damage evolution and lifetime distribution and proposed approximate expressions for the size scaling of coefficient of variation $(\mathrm{COV})$ and mean lifetime. They gave $\mathrm{COV} \approx$ $(\rho / 2-1) / \ln (N)$, where $N$ is the total number of fibers. In this

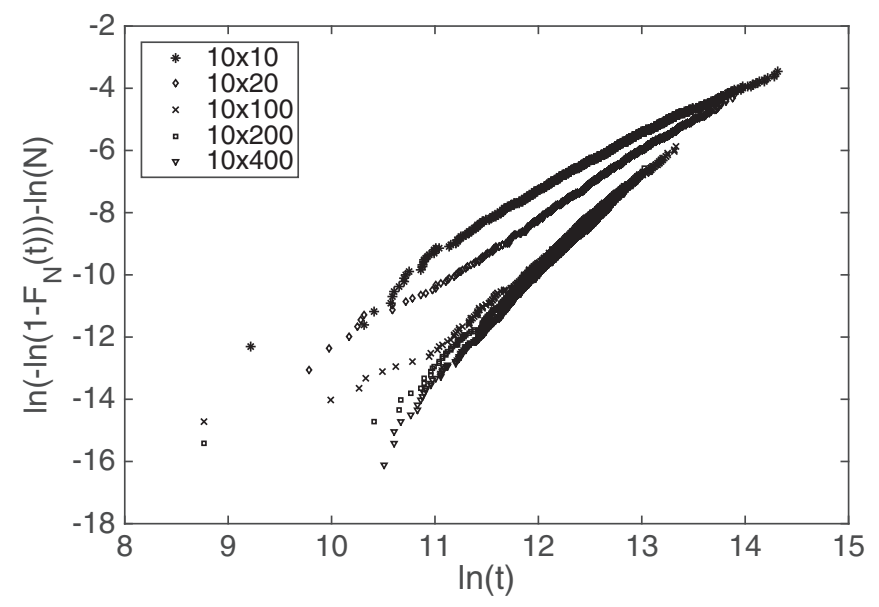

FIG. 6. Lifetime distributions for different sizes of the width of the lattice plotted in a Weibull format with a scaling term.

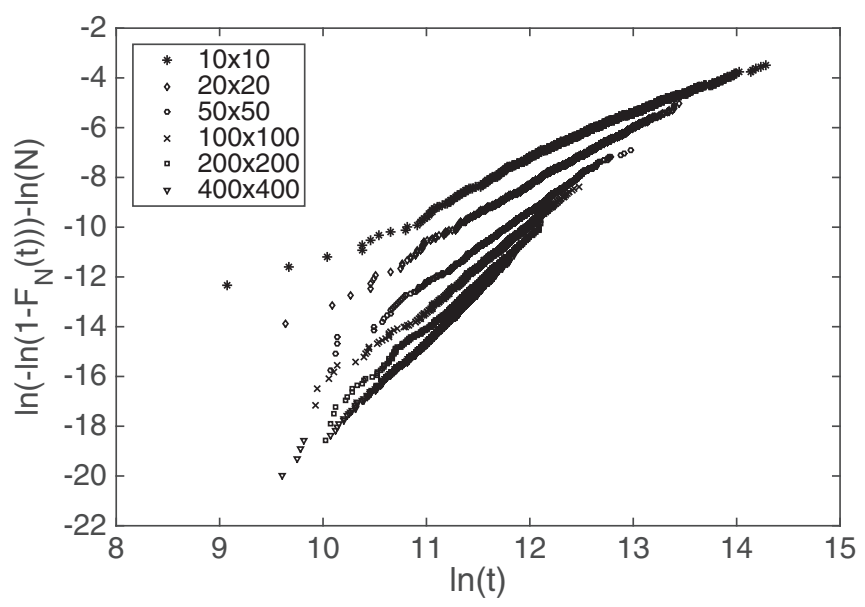

FIG. 7. Lifetime distributions for different sizes of the lattice plotted in a Weibull format with a scaling term.

formula $\mathrm{COV}$ decreases with $N$, and at the thermodynamic limit $(N \rightarrow \infty)$ COV diminishes, although the diminishing rate is slow. Figure 9 shows the relation between $\mathrm{COV}$ and $\ln [\ln (N)]$ obtained from this simulation. The slope was -0.93 , which is very close to -1 as predicted by Curtin and Scher.

The corresponding scaling of mean lifetime is given by $\langle t\rangle \propto(\ln N)^{1-\rho / 2}$. Figure 10 shows the relationship between mean lifetime and $N$ obtained from the simulation.

As can be seen, a linear relationship was found, but the slope was -1.81 , which deviated from the prediction $(1-\rho / 2=$ $-4)$.

These size scaling results pose an interesting but puzzling question. On one hand, as $N$ increases, there was a tendency of weakest-link scaling, and the distribution function at a given size is approximately Weibull distribution. (Precisely saying, it is difficult to detect non-Weibull behavior in a statistically significant manner.) On the other hand, the scaling results for the slope (Weibull shape parameter) and the mean clearly deviate from what is expected from the weakest-link scaling of Weibull distribution (i.e., the constant slope and linear decrease of mean lifetime with $\ln (N)$ [9]). This suggests that the limiting distribution of lifetime as $N \rightarrow \infty$ may not be exactly Weibull

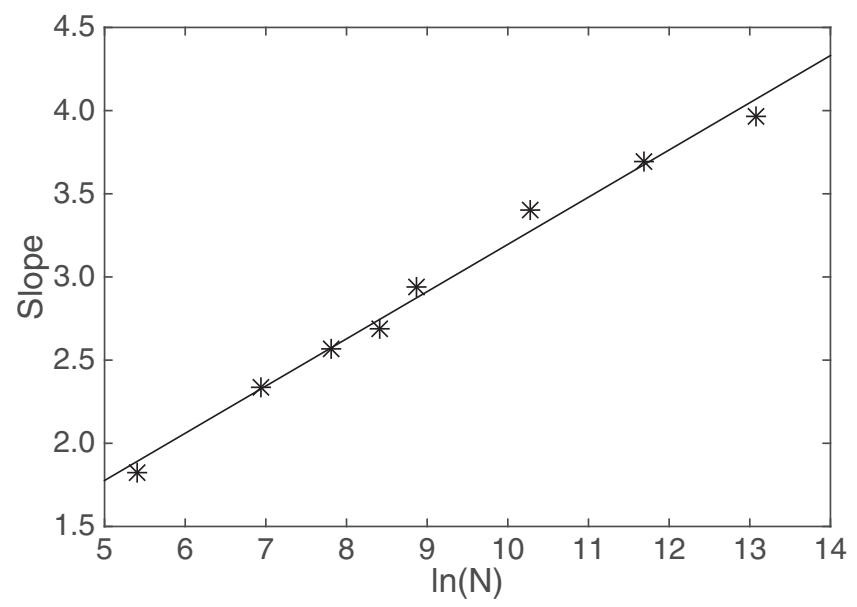

FIG. 8. Slope vs total number of fibers for the lifetime distributions plotted in Fig. 7. 


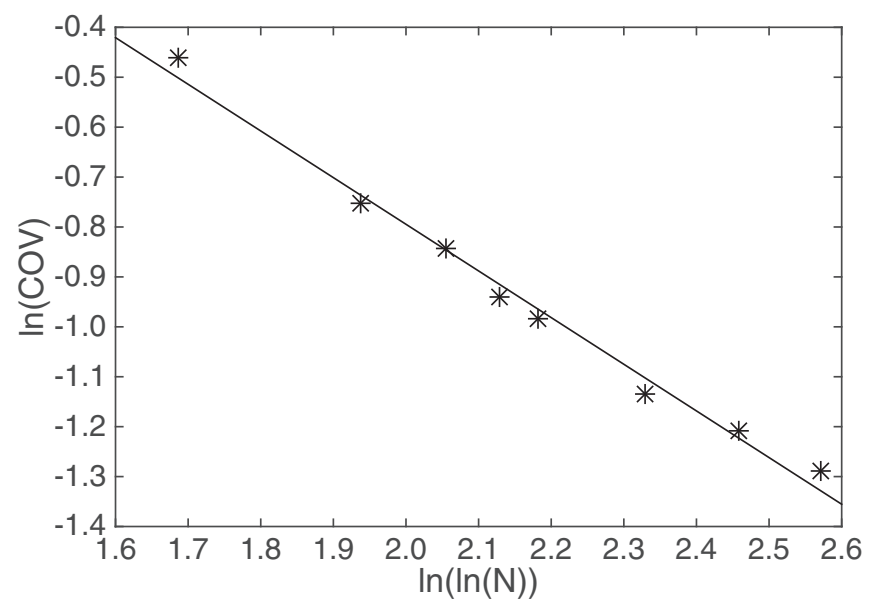

FIG. 9. COV vs total number of fibers for the lifetime distributions plotted in Fig. 7.

distribution. We will further discuss this point in the discussion section. Meanwhile we examine next how lifetime distribution is affected by the failure properties of fibers by using the networks whose size is in the WLS range.

The emergence of weakest-link scaling means that the distribution function for a sufficiently large system size $N$ has this special form:

$$
1-F_{N}(t) \rightarrow[1-W(t)]^{N},
$$

where $W(t)$ is called the characteristic distribution function, representing the collapsed curve when WLS appears [6]. Unlike those curves in a finite-size range (smaller size), $W(t)$ is approximately linear in Weibull plots (Fig. 7). Therefore, we write the approximation of the characteristic distribution function, $W^{*}(t)\left\{\equiv\left[1-F_{N}(t)\right]^{1 / N}\right\}$, as:

$$
\ln \left\{-\ln \left[1-W^{*}(t)\right]\right\} \approx a \ln (t)+b
$$

or

$$
W^{*}(t) \approx 1-\exp \left\{-c t^{a}\right\}
$$

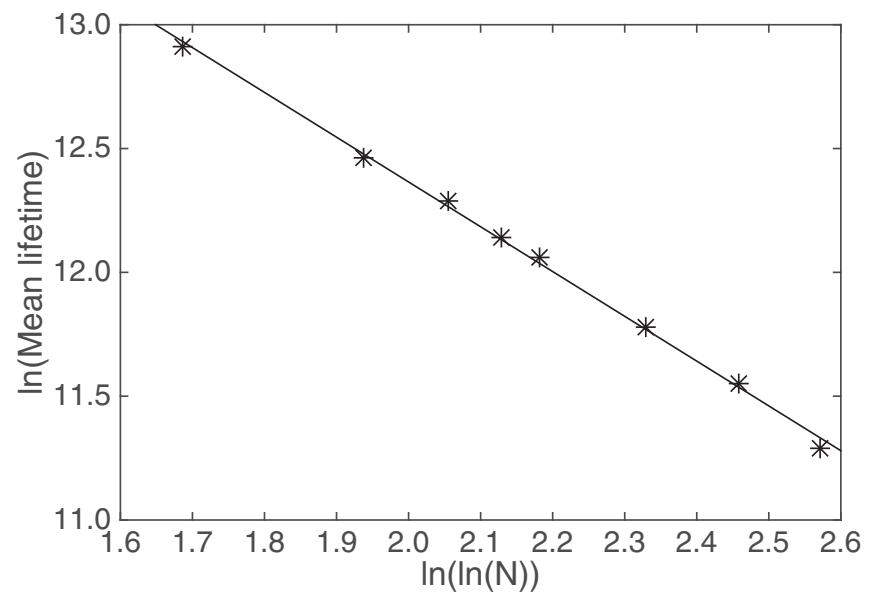

FIG. 10. Mean lifetime vs total number of fibers for the lifetime distributions plotted in Fig. 7. where $c \equiv \exp \{b\}$. The critical questions are, first, whether one can express $W^{*}(t)$ in the form of Eq. (7) that Coleman formulated originally for fiber and, second, how the parameters $a$ and $b$ (or $c$ ) depend on the fiber properties $T_{c}$ and $\rho$.

\section{Effects of applied load}

For numerical simulations, we first find a set of parameters by dimensional consideration. In the case of elastic failure (no geometrical nonlinearity), applied load is linearly related to local force of each fiber $i$ :

$$
T_{i}(t)=S_{0} K_{i}(t)
$$

where $K_{i}(t)$ is stress concentration factor. Using this relation in the lifetime distribution function of fiber [Eq. (9)], we have

$$
F_{i}(t)=1-\exp \left\{-\left(\frac{S_{0}}{T_{c}}\right)^{\rho} \int_{0}^{t} K_{i}(s)^{\rho} d s\right\} .
$$

The system's failure probability is, thus, determined by two nondimensional parameters of fiber, $\left(S_{0} / T_{c}\right)$ and $\rho$, as well as the individual stress (concentration) histories $K_{i}(t)$.

Figure 11 shows the lifetime distributions for different applied loads plotted in Weibull format. The shapes of the distributions are linear, again confirming Weibull distribution. With increasing load, the curves are simply shifted towards shorter lifetime, whereas the slope $a$ (Weibull exponent) remains almost constant with varying load. Therefore,

$$
a=a\left(\frac{S_{0}}{T_{c}}, \rho\right)=a(\rho),
$$

where $a(10)$ varied between 3.09 and 3.65. The intercept $b$ of the Weibull plot is linearly related to applied load, as seen in Fig. 12,

$$
b=a_{1} \ln \left(\frac{S_{0}}{T_{c}}\right)+b_{1} .
$$

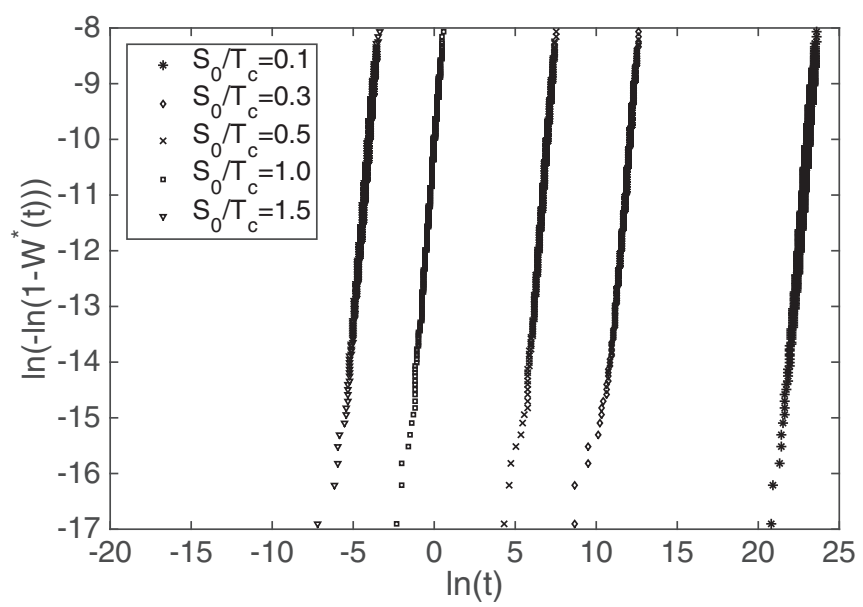

FIG. 11. Lifetime distributions for different applied loads plotted in a Weibull format. 


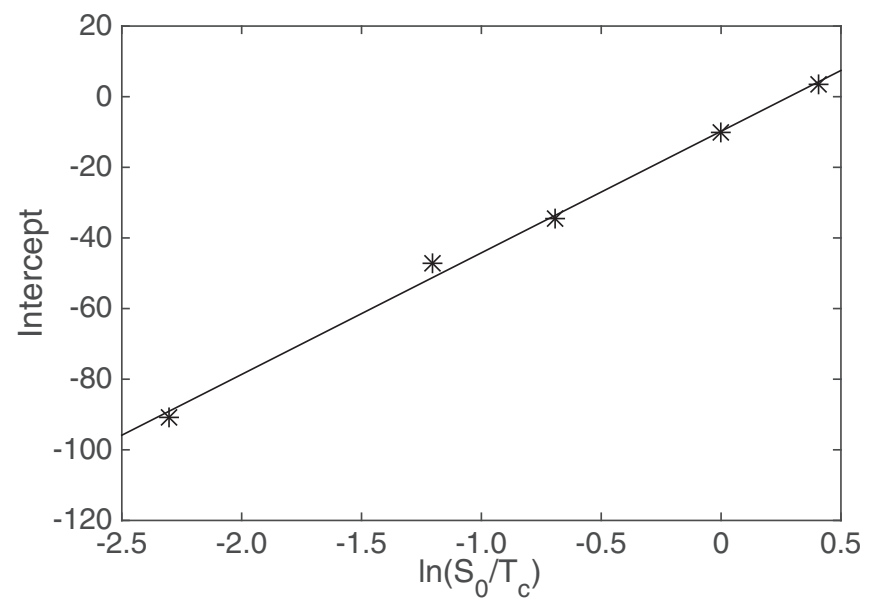

FIG. 12. Intercept vs applied load for the lifetime distributions plotted in Fig. 11.

This indicates that $W^{*}(t)$ is generally expressed as

$$
W^{*}(t)=1-\exp \left\{-c_{1}(\rho)\left(\frac{S_{0}}{T_{c}}\right)^{a_{1}(\rho)} t^{a(\rho)}\right\},
$$

where $c_{1}\left(=\exp \left\{b_{1}\right\}\right), a_{1}$, and $a$ generally depend on $\rho$. From Eq. (14), the dependence on $S_{0} / T_{c}$ must be in the form of $\left(S_{0} / T_{c}\right)^{\rho}$. This means that $a_{1}(\rho)=a_{2} \rho$, where $a_{2}$ may still depend on $\rho$ at this stage.

\section{Effects of load sensitivity factor $\rho$}

Figure 13 shows lifetime distributions when load sensitivity factor $\rho$ is varied. As $\rho$ increases, the lifetime distributions shift towards longer lifetimes at the same time as the slope decreases (i.e., more variation of lifetime).

In Fig. 14, the slope of the distributions is plotted against different values of $\rho$. It is interesting to see that, as $\rho$ increases, the slope (Weibull shape parameter) decreases but reaches a plateau value, unity, which happens to be the value of Weibull shape parameter of fiber. In other words, the network's reliability may be ultimately controlled by fiber at

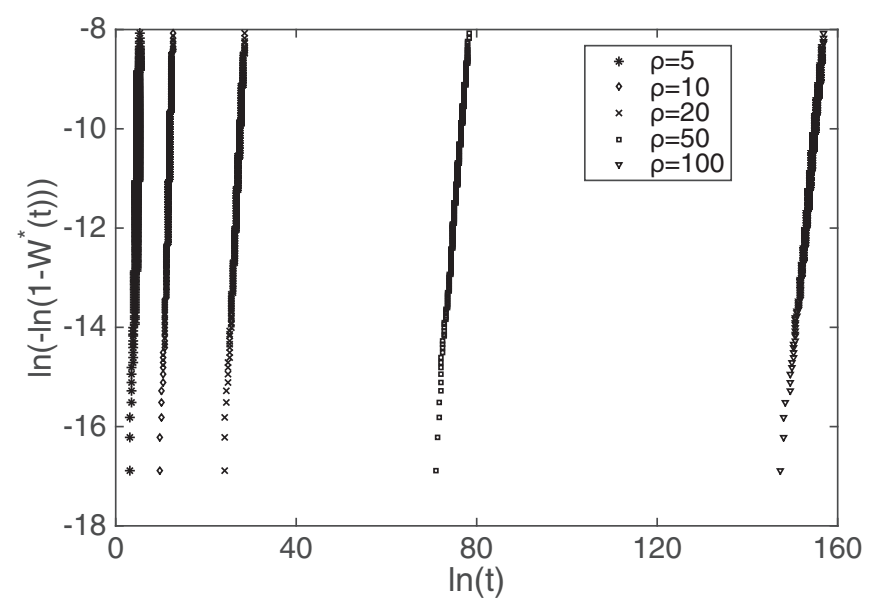

FIG. 13. Lifetime distributions for different values of load sensitivity factor, $\rho$, plotted in a Weibull format.

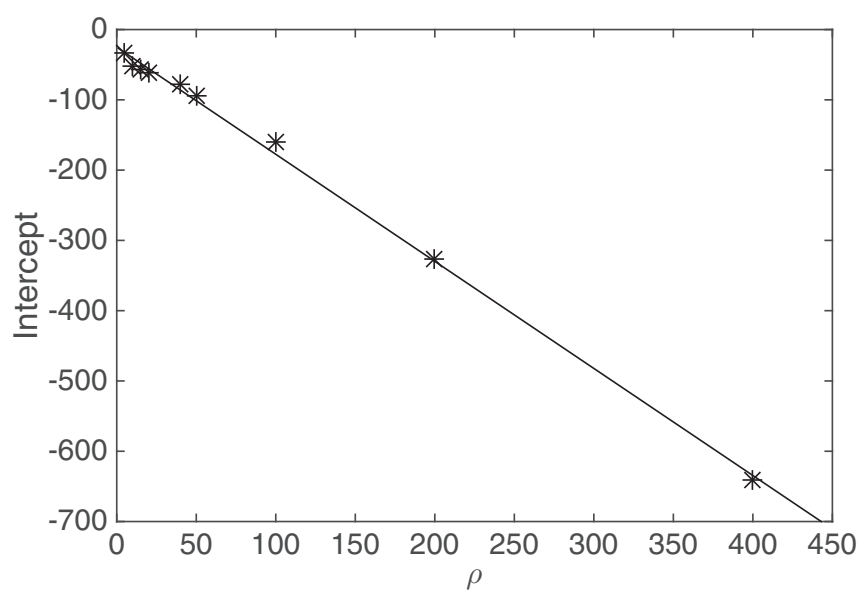

FIG. 14. Slope vs load sensitivity factor, $\rho$, of the lifetime distributions plotted in Fig. 13.

high $\rho$ values. Figure 14 also determines the dependence of $a(\rho)$ on $\rho$ in Eq. (17).

In Fig. 15, the intercept for the distributions is plotted against different values of $\rho$. The intercept clearly shows a linear relation with $\rho$. Using Eq. (17), we have the following equality:

$$
b=a_{3} \rho+b_{3}=\ln \left[c_{1}(\rho)\right]+a_{2}(\rho) \rho \ln \left(\frac{S_{0}}{T_{c}}\right) .
$$

where the slope $a_{3}$ and the intercept $b_{3}$ in Fig. 15 are generally a function of $S_{0} / T_{c}$. In order to maintain the linear form on the right-hand side, $c_{1}(\rho)$ must be expressed as $C_{a} C_{b}^{\rho}$, and $a_{2}(\rho)$ must be a constant (instead of a function of $\rho$ ). This eventually determines the functional form of $W^{*}$, which is given by

$$
W^{*}(t)=1-\exp \left\{-C_{a} C_{b}^{\rho}\left(\frac{S_{0}}{T_{c}}\right)^{a_{2} \rho} t^{a(\rho)}\right\} .
$$

This is further transformed into a more compact form:

$$
W^{*}(t)=1-\exp \left\{-\left(\frac{S_{0}}{S_{c}^{*}}\right)^{\rho^{*} \beta^{*}} t^{\beta^{*}}\right\},
$$

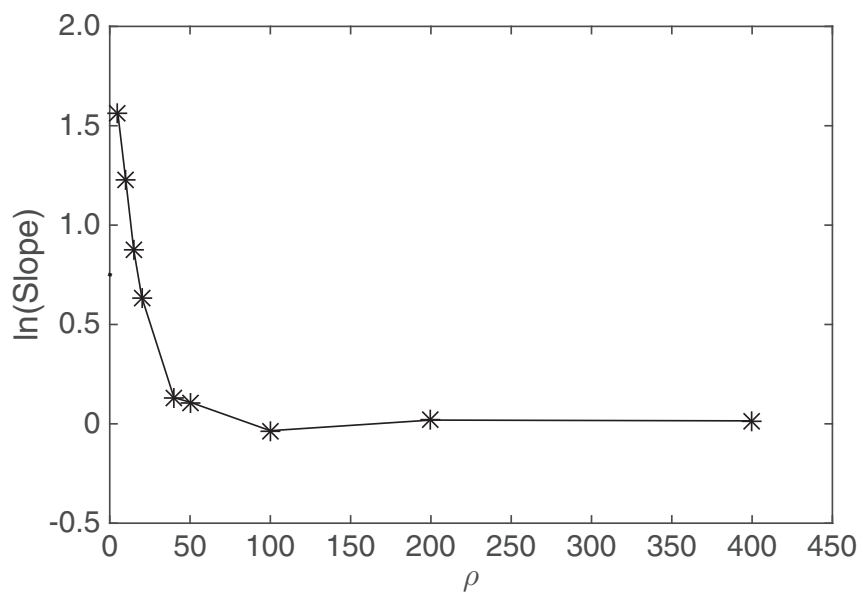

FIG. 15. Intercept vs load sensitivity factor, $\rho$, of the lifetime distributions plotted in Fig. 13. 


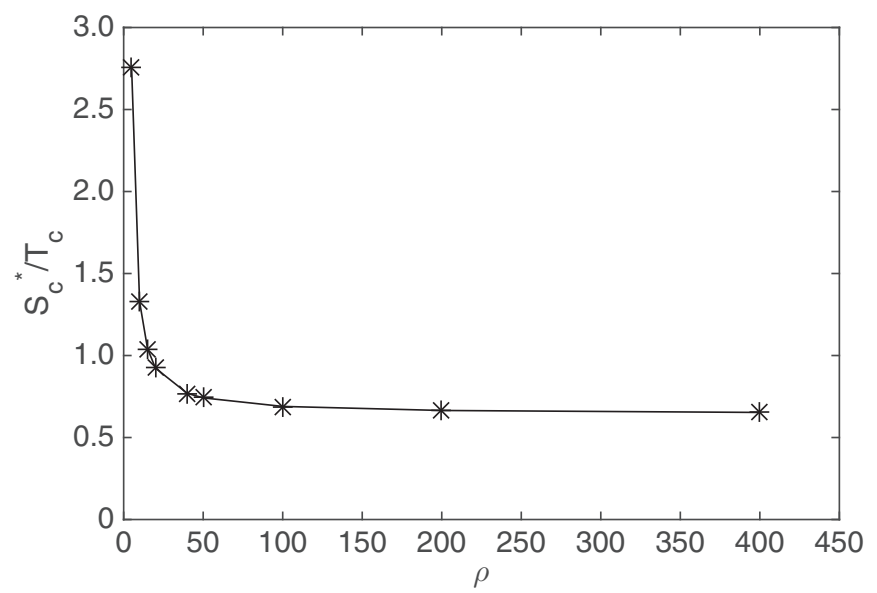

FIG. 16. Nondimensional characteristic strength $S_{c}^{*} / T_{c}$ of the network vs fiber $\rho$.

where $S_{c}^{*} / T_{c}=\left(C_{a} C_{b}^{\rho}\right)^{-1 /\left(a_{2} \rho\right)}, \rho^{*}=\left[a_{2} \rho / a(\rho)\right]$, and $\beta^{*}=$ $a(\rho)$. The corresponding expression for fiber is given by

$$
F_{f}(t)=1-\exp \left\{-\left(\frac{T_{0}}{T_{c}}\right)^{\rho \beta} t^{\beta}\right\},
$$

where $\beta$ is assumed to be unity in the simulation. When comparing the above two equations, we note that the parameters $S_{c}^{*}, \rho^{*}$, and $\beta^{*}$ are those for a fictitious elements of the network. Here in Eq. (20) we have recovered Coleman's formula for the cumulative distribution function of creep lifetime on the system level. The preservation of Coleman's formula when ascending the structural hierarchy from fiber to fiber network may not be completely surprising. It is because we used the power-law breaking law (scale-free) and also the deformation is still in a geometrically linear range (small strain). This preservation property was also indicated by Phoenix and Tierney in the case of a fiber bundle model [11].

Figure 16 shows the effect of $\rho$ of fiber on the nondimensional characteristic strength of the fictitious elements $S_{c}^{*} / T_{c}$. With increasing $\rho, S_{c}^{*}$ quickly decreased and reached a plateau below $T_{c}$, fiber characteristic strength. It is interesting to see that $S_{c}^{*}$ is even higher than $T_{c}$ in the low $\rho$ range. Figures 17 and 18 show the effects of $\rho$ of fiber on load sensitivity factor

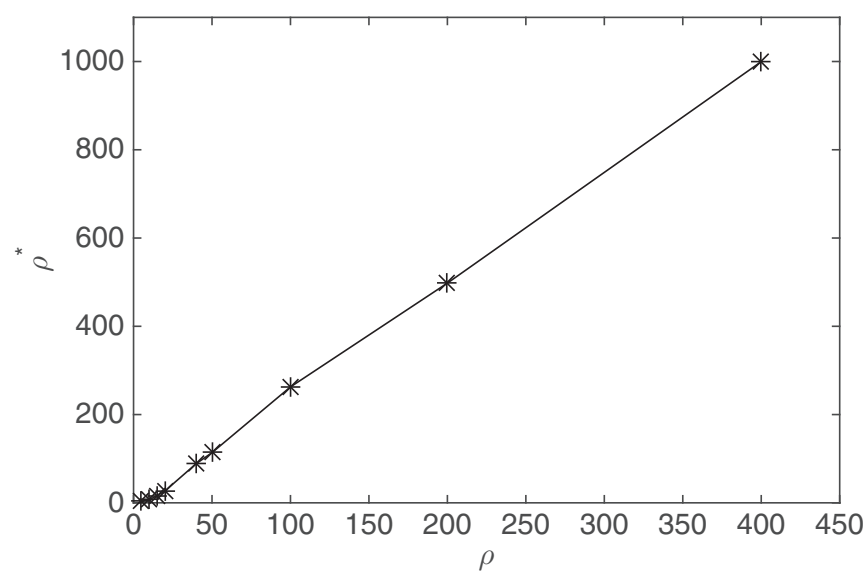

FIG. 17. Load sensitivity factor of the network, $\rho^{*}$ vs fiber $\rho$.

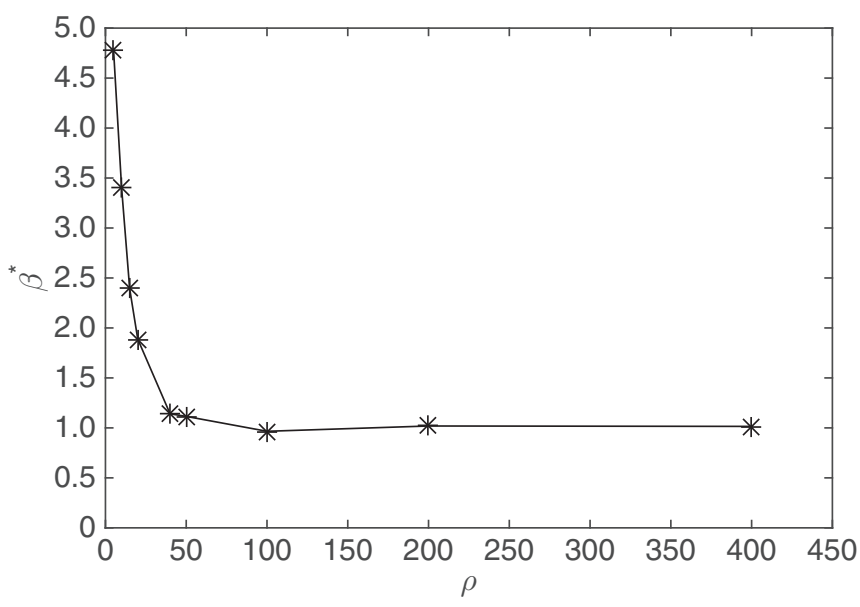

FIG. 18. Weibull shape parameter of the network, $\beta^{*}$ vs fiber $\rho$.

of the network, $\rho^{*}$, and the corresponding Weibull shape parameter $\beta^{*}$. Increasing $\rho$ increased $\rho^{*}$ and decreased $\beta^{*}$, and $\beta^{*}$ approaches to unity, as discussed earlier.

\section{E. Effects of fiber strength, $T_{c}$}

The system investigated so far contains fibers whose failure property follows Coleman's probabilistic failure law that is characterized by the parameters, $T_{c}, \rho$, and $\beta(=1)$. In a heterogeneous network, these parameters may vary from one fiber to the other, and thus the statistics are superimposed (superstatistics). In this section, we have varied the characteristic strength, $T_{c}$, to simulate the effects of heterogeneous fibers in the network on the lifetime distribution.

Figure 19 shows lifetime distributions when $T_{c}$ is generated as a uniformly distributed random variable between $T_{c \text {,min }}$ and $T_{c, \max }$, where we fixed the mean (10) but varied $\Delta T_{c}$ $\left(=T_{c, \text { max }}-T_{c, \text { min }}\right)$. As can be seen, when increasing the spread of fiber characteristic strength, the distributions shift towards shorter lifetimes but, surprisingly, retain Weibull distribution. Figure 20 shows the slopes (Weibull shape parameter) of the distributions as a function of the spread $\Delta T_{c} / T_{c}$. The

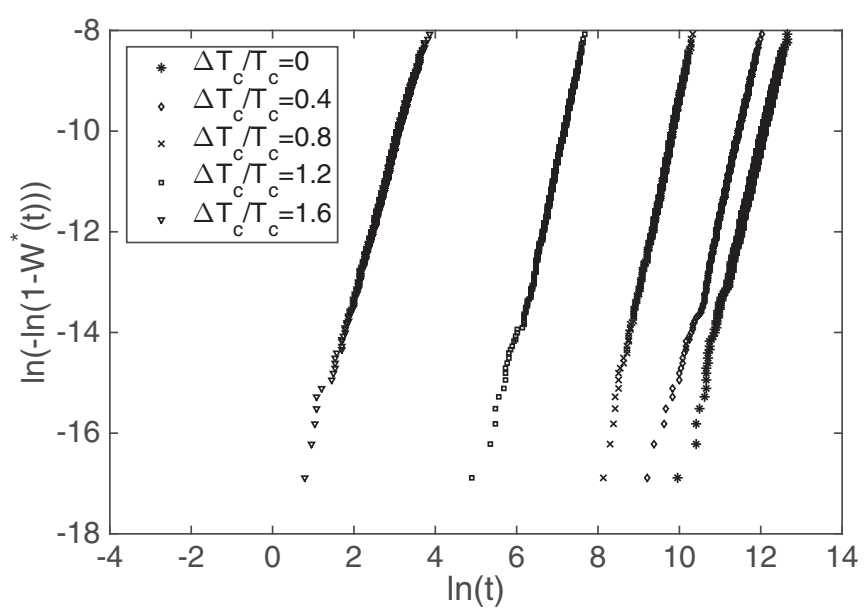

FIG. 19. Lifetime distributions for different values of the spread of characteristic strength of fiber, $\Delta T_{c} / T_{c}$, plotted in a Weibull format. 


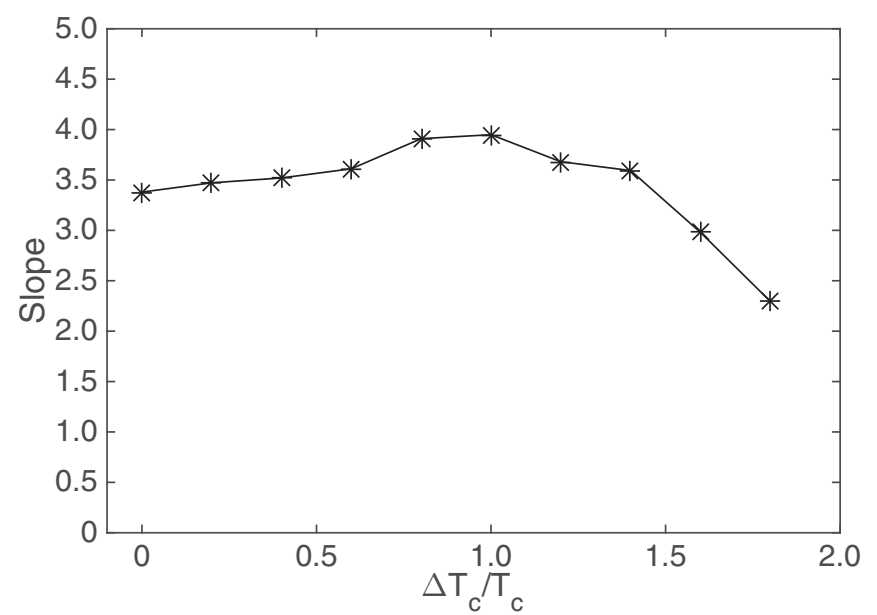

FIG. 20. Slope vs spread of characteristic strength of fiber, $\Delta T_{c} / T_{c}$, for the lifetime distributions plotted in Fig. 19 .

slope gradually increases with the spread but decreases as $\Delta T_{c} / T_{c} \rightarrow 2$. It is difficult to surmise the underlying mechanism, but it may be related to the non-dimensional load parameter, $\left[T(t) / T_{c}\right]^{\rho}$ in Eq. (9). As the spread increases, this parameter increases (i.e., increased failure probability) at the lower end, $T_{c}=T_{c, \text { min }}$, whereas it decreases at another end $\left(T_{c}=T_{c, \max }\right)$. Therefore, the phenomena seen in Fig. 20 may be a result of these two competing processes.

\section{F. Effects of stress distributions}

In addition to the fiber properties, another superimposed statistics may be stress (force per fiber) distribution. The triangular lattice that we used has its own stress distribution according to its geometry, but we can also introduce additional stress disorder by manipulating some of the structural parameters in the triangular lattice model. For this purpose we varied the area $A$ of the fibers (truss elements) by generating uniformly distributed random variables between $A_{\text {min }}$ and $A_{\text {max }}$. This creates additional stress disorders to this triangular network.

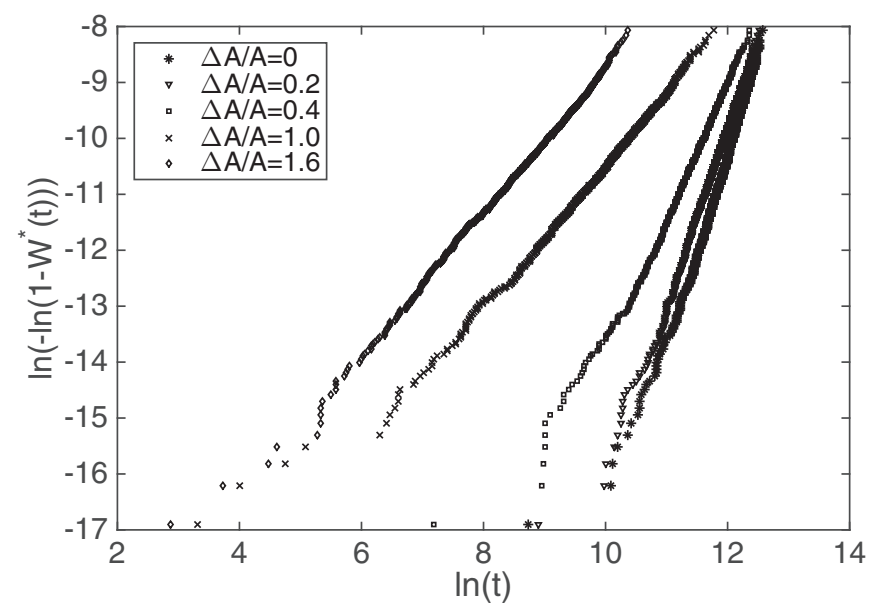

FIG. 21. Lifetime distributions for different values of $\Delta A / A$ plotted in a Weibull format.

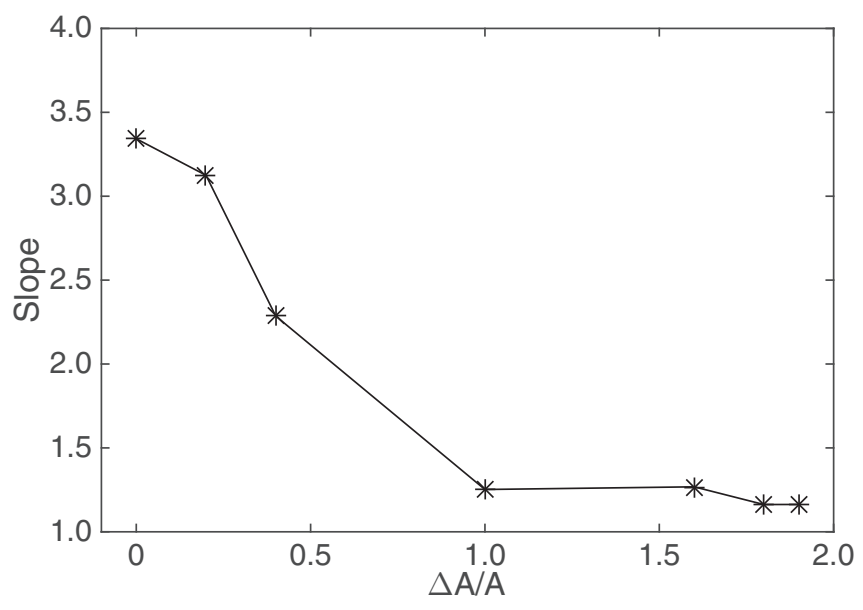

FIG. 22. Slope vs $\Delta A / A$ for the lifetime distributions plotted in Fig. 21.

Figure 21 shows the lifetime distributions for different spreads $\triangle A / A$. Increasing the spread shifts the distribution towards shorter lifetimes, and at the same time the slope decreases. This decrease of the slope eventually ceased (Fig. 22) and approached unity, which is, again, the Weibull shape parameter of fiber. In other words, the stress disorder decreases both the mean lifetime and the shape parameter of the network. For large stress disorders in the network fiber's uncertainty of lifetime seems to ultimately control the one for the network.

\section{DISCUSSIONS}

Although Coleman's phenomenological model was originally developed as a model for time-dependent statistical failure of fiber, it is interesting to see that the same probabilistic failure law appeared also on the network level. From the point of Coleman's formulation, however, it is expected that such a relationship holds as long as the material system satisfies the three conditions that Coleman postulated: (1) WLS, (2) power-law form of probabilistic failure criteria (which leads to Weibull distribution of creep lifetime), and (3) powerlaw form of damage evolution with load.

Weakest-link scaling is associated with brittle or quasibrittle failure. In the system we have studied with $\rho>2$, it is, therefore, reasonable to observe WLS as a limiting case when the total number of (fictitious) elements becomes large.

One interesting result is that, although WLS seems to emerge for this network system and the distribution at a given network size is approximately Weibull distribution, the scaling behavior of the characteristic strength (and mean strength) and the slope did not follow the WLS for Weibull distribution. Instead they followed:

$$
\begin{aligned}
\beta_{N} & =a_{\beta} \ln (N)+b_{\beta}, \\
\ln \left(\alpha_{N}\right) & =-a_{\alpha} \ln [\ln (N)]+b_{\alpha},
\end{aligned}
$$


where $\beta_{N}$ and $\alpha_{N}$ are defined in the approximation of lifetime distribution at size $N$ :

$$
1-F_{N}(t) \simeq \exp \left[-\left(\frac{t}{\alpha_{N}}\right)^{\beta_{N}}\right] .
$$

The question is whether there is a function $W(t)$ that satisfies the weakest-link scaling $\left\{1-F_{N}(t) \rightarrow[1-W(t)]^{N}\right.$ as $N \rightarrow \infty\}$, and, at the same time, produces the scaling relations in Eq. (22). In fact there is such a function when we consider $W(t)$ as an envelope that is formed by a series of the tangent curves, each of which is described by Eq. (23). This is actually a similar conjecture that was also used by Phoenix and Tierney [11] and Newman and Phoenix [6] to approximate $W(t)$ by the critical cluster model of LLS fiber bundles. As shown in Appendix, $W(t)$ is given by

$$
W(t)=1-\exp \left\{-\exp \left\{-\lambda t^{-1 / a_{\alpha}}+\mu\right\}\right\}
$$

or

$$
F_{N}(t)=1-\exp \left\{-N \exp \left\{-\lambda t^{-1 / a_{\alpha}}+\mu\right\}\right\}
$$

where

$$
\begin{aligned}
& \lambda=a_{\alpha} a_{\beta} \exp \left\{\frac{1}{a_{\alpha} a_{\beta}}+\frac{b_{\alpha}}{a_{\alpha}}-1\right\}, \\
& \mu=b_{\beta}\left(\frac{1}{a_{\beta}}-a_{\alpha}\right) .
\end{aligned}
$$

This equation is valid for small $t$ (or large $N$ ), as seen in the Appendix. Although this is still an empirical result based on the simulations, it is interesting to see that $W(t)$ or $F_{N}(t)$ has a double exponential form, which was also obtained by Curtin and Scher from their damage evolution model [23].

One important note is that this model does not take into account the dynamic effect of cracking (or avalanche). The presence of dynamic effects was recently demonstrated for a triangular lattice model with distributed strength thresholds [36]. The study showed that a single bond break can cause immediate multiple breaks by the dynamic effect. Therefore, the finite time required to proceed from avalanche to the total failure, such as observed in many static fiber bundle models, may be an overestimate.

The parameters defined in Eq. (20), $S_{c}^{*}, \rho^{*}$, and $\beta^{*}$, are useful for material characterization in terms of timedependent, statistical failure. One interesting observation in this study is that at the brittle limit $(\rho \rightarrow \infty)$, the Weibull shape parameter of the system $\beta^{*}$ has a lower bound which seems to coincide with the same shape parameter of fiber. This is understandable, since at the brittle limit, the lifetime of the system is essentially determined by the first fiber failure, which is simply a minimum of lifetime among all fibers. In other words, suppose the lifetime distribution of the $i$-th fiber is given by

$$
F_{i}(t)=1-\exp \left(-\left\{\int_{s=0}^{t}\left[\frac{T_{i}(s)}{T_{c}}\right]^{\rho} d s\right\}^{\beta}\right),
$$

then lifetime distribution of the system [i.e., the minimum of lifetime of $\mathrm{N}$ fibers $F_{N}(t)$ ] should satisfy the condition:

$$
\begin{aligned}
1-F_{N}(t) & =\prod_{i=1}^{N}\left[1-F_{i}(t)\right] \\
& =\exp \left(-\left\{\sum_{i=1}^{N}\left[\frac{T_{i}(0)}{T_{c}}\right]^{\rho \beta}\right\} t^{\beta}\right),
\end{aligned}
$$

where $T_{i}(0)$ is the force applied to the $i$-th fiber between $t=0$ and the first fiber failure. Therefore, lifetime distribution of the system for the first fiber failure is Weibull distribution with the shape parameter being equal to the shape parameter of fiber $\beta$. Note that this result is not limited to a specific geometry; it is equally applied to a $3 \mathrm{D}$ fiber network at $\rho \rightarrow \infty$.

\section{SUMMARY AND CONCLUDING REMARKS}

Numerical simulations of creep failure have been carried out for central-force, triangular fiber networks by assuming element fibers follow Coleman's probabilistic failure law, with $\beta=1$ and $\rho>2$. The main results obtained are as follows:

First, Coleman's probabilistic failure law approximately holds also on the system level (fiber network) when the network size enters into the WLS regime. In this regime, Weibull distribution was persistent even when there are superimposed disorders to fiber properties or stress distributions. However, Weibull distribution is still an approximation of $W(t)$, which was actually found to have a double exponential form rather than Weibull form.

Second, comparing fiber properties $T_{c}, \rho, \beta$ with those for the network $S_{c}^{*}, \rho^{*}, \beta^{*}$, we found that increasing fiber $\rho$ induces a reduction of characteristic strength $S_{c}^{*}$ but an increase of load sensitivity $\rho^{*}$ and Weibull shape parameter $\beta^{*}$ of the network. The superimposed disorders of fiber characteristic strength and stress reduces both characteristic strength and $\beta^{*}$ of the network significantly.

\section{ACKNOWLEDGMENT}

The authors thank the KK-foundation for financial support through their KK-environment program and SCA R\&D and BillerudKorsns for their supports for the project, Light-weight structural composites, in which this work is conducted. We also thank Dr. Johan Persson for valuable comments and suggestions made during the development of the simulation code for this work.

\section{APPENDIX}

\section{Damage evolution under Kachanov's type condition}

Coleman's breakdown rule of Class B states that the damage evolution depends not only on force applied on the fiber but also the damage state itself, which is expressed as

$$
\frac{d \Omega}{d t}=\phi[f(t), \Omega(t)]
$$


One of the examples of Coleman's Class B damage evolution rule is the one by Kachanov [28]:

$$
\frac{d \Omega_{n}}{d t}=B\left[\frac{f(t)}{1-\Omega_{n}(t)}\right]^{\rho},
$$

where $\Omega_{n}$ is a normalized damage variable, according to Kachanov, with $\Omega_{n}=\Omega / \Omega_{0}$ and $\Omega_{0}$ the damage level at which the stress enhancement effect becomes infinity. The above equation can be easily integrated:

$$
\frac{1}{\rho+1}\left[1-\left(1-\Omega_{n}\right)^{\rho+1}\right]=B \int_{0}^{t} f(s)^{\rho} d s .
$$

For $\Omega \ll 1$, i.e., when failure happens with a limited level of damages (brittle failure), then the left-hand side is approximated as

$$
\Omega_{n}+O\left(\Omega_{n}^{2}\right)=B \int_{0}^{t} f(s)^{\rho} d s .
$$

This recovers Coleman's Class A breaking rule. In other words, the assumption of the Class A breaking rule implicitly contains the assumption of brittle failure, which goes together with the case of higher $\rho$ failures. Equation (A3) can be solved for $\Omega_{n}$ as

$$
\Omega_{n}=1-\left[1-B(\rho+1) \int_{0}^{t} f(s)^{\rho} d s\right]^{\frac{1}{\rho+1}} .
$$

This damage evolution, based on Kachanov's type condition, clearly gives non-Weibull distribution of creep lifetime.

\section{Characteristic distribution function}

Weakest-link scaling assumes the presence of characteristic distribution function $W(t)$, such that

$$
1-F_{N}(t) \rightarrow[1-W(t)]^{N}
$$

as $N \rightarrow \infty$. In this simulation we have found that $F_{N}(t)$ is approximated by Weibull distribution within the probability range tested (0.001 and 0.999):

$$
1-F_{N}(t) \simeq \exp \left[-\left(\frac{t}{\alpha_{N}}\right)^{\beta_{N}}\right]
$$

and $\alpha_{N}$ and $\beta_{N}$ were scaled by

$$
\begin{aligned}
\beta_{N} & =a_{\beta} \ln (N)+b_{\beta}, \\
\ln \left(\alpha_{N}\right) & =-a_{\alpha} \ln [\ln (N)]+b_{\alpha} .
\end{aligned}
$$

The question is how to find $W(t)$ that satisfies the scaling relations in Eq. (A6) and Eq. (A8), as $N \rightarrow \infty$. As seen in Fig. 7, we can consider $W(t)$ as an envelope to which a series of the approximate curves Eq. (A2) for different $N$ form tangents. This is the similar conjecture that was employed by Phoenix and Tierney and Newman and Phoenix for approximating $W(t)$ by using the lifetime distributions of critical damage clusters in fiber bundle model with local-load sharing [6,11]. From Eq. (A6), if $\left[1-F_{k}(t)\right]^{1 / k}$ form tangents to $1-W(t)$, we must be able to find $t_{k}$ which satisfies

$$
\left[1-F_{k}\left(t_{k}\right)\right]^{1 / k}=\left[1-F_{k+1}\left(t_{k}\right)\right]^{1 /(k+1)} .
$$

This relation yields

$$
\ln \left(t_{N}\right)=a_{1}-a_{\alpha} \ln [\ln (N)]-\frac{a_{2}}{\ln (N)},
$$

where $a_{1}=1 / a_{\beta}-a_{\alpha}+b_{\alpha}$ and $a_{2}=a_{\alpha} b_{\beta} / a_{\beta}$. Using Eq. (A10) in Eqs. (A6) and (A7), and taking $N \rightarrow \infty$, we have

$$
W(t)=1-\exp \left\{-\exp \left\{-\lambda t^{-1 / a_{\alpha}}+\mu\right\}\right\},
$$

where

$$
\begin{aligned}
\lambda & =a_{\alpha} a_{\beta} \exp \left\{\frac{1}{a_{\alpha} a_{\beta}}+\frac{b_{\alpha}}{a_{\alpha}}-1\right\}, \\
\mu & =b_{\beta}\left(\frac{1}{a_{\beta}}-a_{\alpha}\right) .
\end{aligned}
$$

This double exponential form is an approximation when $N \rightarrow$ $\infty$ (or $t \rightarrow 0)$.
[1] A. Mattsson and T. Uesaka, in Transactions of the 15th Fundamental Research Symposium, Vol. 1, edited by S. I'Anson (Cambridge University Press, Cambridge, 2013), pp. 711-734.

[2] S. L. Phoenix, Int. J. Fract. 14, 327 (1978).

[3] H. Otani, S. L. Phoenix, and P. Petrina, J. Mater. Sci. 26, 1955 (1991).

[4] H. Wu, S. L. Phoenix, and P. Schwartz, J. Mater. Sci. 23, 1851 (1988).

[5] W. Curtin, Adv. Appl. Mech. 36, 163 (1999).

[6] W. I. Newman and S. L. Phoenix, Phys. Rev. E 63, 021507 (2001).

[7] Z. Bazãnt, Proc. Natl. Acad. Sci. USA 101, 13400 (2004).

[8] B. D. Coleman, J. Appl. Phys. 28, 1058 (1957).

[9] B. D. Coleman, J. Appl. Phys. 29, 968 (1958).

[10] S. L. Phoenix, Adv. Appl. Probab. 11, 153 (1979).

[11] S. L. Phoenix and L. Tierney, Eng. Fract. Mech. 18, 193 (1983).
[12] L. Tierney, Adv. Appl. Probab. 14, 95 (1982).

[13] T. Uesaka and J. Juntunen, Nord. Pulp Paper Res. J. 27, 370 (2012).

[14] S. Mahesh and S. L. Phoenix, Int. J. Fract. 127, 303 (2004).

[15] M. Alava, P. Nukala, and S. Zapperi, Adv. Phys. 55, 349 (2006).

[16] H. E. Daniels, Proc. R. Soc. London A 183, 404 (1945).

[17] B. D. Coleman, J. Mech. Phys. Solids 7, 60 (1958).

[18] R. P. Kelley, Further examination and a local load-sharing extension of Coleman's time to failure model for fiber bundles, Ph.D. thesis, Cornell University (1978).

[19] W. Newman and A. Gabrielov, Int. J. Fract. 50, 1 (1991).

[20] W. I. Newman, A. M. Gabrielov, T. A. Durand, S. L. Phoenix, and D. L. Turcotte, Physica D 77, 200 (1994).

[21] B. D. Coleman, J. Polymer Sci. 20, 447 (1956).

[22] D. T. Hristopulos, M. P. Petrakis, and G. Kaniadakis, Phys. Rev. E 89, 052142 (2014). 
[23] W. A. Curtin and H. Scher, Phys. Rev. B 55, 12038 (1997).

[24] H. D. Wagner, P. Schwartz, and S. L. Phoenix, J. Mater. Sci. 21, 1868 (1986).

[25] C. M. Landis, I. J. Beyerlein, and R. M. McMeeking, J. Mech. Phys. Solids 48, 621 (2000).

[26] W. A. Curtin, M. Pamel, and H. Scher, Phys. Rev. B 55, 12051 (1997).

[27] M. Vujosevic and D. Krajcinovic, Int. J. Solids Struct. 34, 1105 (1997).

[28] L. Kachanov, Introduction to Continuum Damage Mechanics, Mechanics of Elastic Stability (Martinus Nijhoff, Lancaster, UK, 1986).

[29] W. A. Curtin and H. Scher, Phys. Rev. B 45, 2620 (1992).
[30] P. M. Duxbury, S. Kim, and P. L. Leath, Mater. Sci. Eng. A176, 25 (1994).

[31] P. M. Duxbury and P. L. Leath, Phys. Rev. B 49, 12676 (1994).

[32] W. A. Curtin and H. Scher, J. Mater. Res. 5, 535 (1990).

[33] H. Rahami, Truss Analysis (2007), http://www.mathworks.com/ matlabcentral/fileexchange/14313-truss-analysis/ (accessed May 28, 2015).

[34] D. T. Hristopulos and T. Uesaka, Phys. Rev. B 70, 064108 (2004).

[35] S. Heyden, Network Modelling for Evaluation of Mechanical Properties of Cellulose Fibre Fluff, Vol. 1011 (Lund University Press, Lund, Sweden, 2000).

[36] G. Villalobos, F. Kun, and J. D. Muñoz, Phys. Rev. E 84, 041114 (2011). 